\title{
Optimal Solution for Localization of a Target Using RSS-AOA Measurements
}

\author{
Hyukwoo Lee, Kyunghyun Lee, and Kwanho You
}

\begin{abstract}
In many industrial areas, the localization method using the active radio signal is widely used due to its reliability under indoor environment compared with global positioning system (GPS). Received signal strength (RSS) and angle of arrival (AOA) measurement based localization is a commonly used scheme for location estimation using active radio source from a target. In this paper, we propose the hybrid localization method using RSS and AOA measurement that has advantages of each localization scheme. However, the measurement noise in RSS and AOA data causes the estimation inaccuracy of a target location. In order to improve the localization accuracy, Newton method is applied to hybrid localization model based objective function that denotes the amount of estimation error. The optimal solution of a target location is acquired through the iteration process based on hybrid localization model and Newton method. Some simulation results demonstrate the effectiveness of our proposed scheme.
\end{abstract}

Index Terms - Localization, received signal strength, angle of arrival, optimization, Newton method.

\section{INTRODUCTION}

The localization method using an active radio signal has received great interest in accordance with the development of location based services such as augmented/virtual reality platform, mobile application, and internet of things. Although recent GPS technology shows the high localization performance, there is an accuracy limitation for indoor or urban environment localization due to jamming. Recently, the localization methods using the object that transmits the active radio signal such as time of arrival (TOA), time difference of arrival (TDOA), and frequency difference of arrival (FDOA) are widely used [1]-[3]. The location of a target can be estimated using the various characteristics of the active radio signal transmission. Moreover, the active radio signal based localizations are almost free from spatial constraints since the base station that receives the radio signal from a target can be arranged freely.

In many localization methods, RSS and AOA based localizations are widely used due to their location estimation accuracy and efficiency. The RSS based localization uses the trilateration approach in which the distance between a target and base station is determined by measured propagation path losses of radio signal. The AOA based localization uses the measurement angle data of signal incidence on an antenna array from base station [4], [5]. Although the RSS and AOA based localization determines relatively accurate location of a

Manuscript received August 3, 2018; revised December 12, 2018.

The authors are with the department of electrical and computer engineering, Sungkyunkwan University, Suwon, Korea (e-mail: lhw2065@skku.edu, naman2001@skku.edu, khyou@skku.edu). target compared to GPS, there exist the noise factors such as signal disruption and interference.

The measurement noise problem that causes the estimation inaccuracy of a target location is the most significant issue in localization. To improve the localization accuracy, Nerguizian [6] proposed a fingerprinting technique using the channel's impulse response information in conjunction with an artificial neural network. The proposed fingerprinting technique used for the geolocation of the studied mine gave an accurate mobile station location. Wang [7] proposed the Monte Carlo importance sampling technique in order to find an approximate global solution of localization problem. Moreover, the efficient importance function was obtained by the Gaussian distribution and probability density function. Jung [8] suggested the indoor localization using TDOA measurements through LED ceiling lamps. A unique frequency address that is assigned to each LED lamp was used to identify each signal.

In this paper, the hybrid localization model using both RSS and AOA measurements is formulated. The hybrid scheme can improve localization performance and reduce the required number of base station. Moreover, we apply the Newton method to hybrid model for the minimization of estimation error. Newton method uses the first and the second derivative function of the objective function to find the optimal solution. The organization of this paper is as follows. In Section II, the localization models using RSS and AOA measurements are derived separately. With the combination of each localization model, the hybrid model is acquired as a simple matrix form. Section III derives the objective function that denotes the amount of estimation error based on hybrid localization model. Moreover, Newton method based iteration process is applied to objective function. Newton method obtains the optimal solution of location estimation with the minimization of estimation error. Some simulations show the localization performance of our proposed method in Section IV. Finally, conclusion is expressed in Section V.

\section{LOCALIZATION USING RSS-AOA MEASUREMENTS}

Hybrid localization using different types of the measured wireless signal data is an efficient method for estimation of a target location. Location estimation method using at least two measurements has advantages such as high localization accuracy. Moreover, localization is feasible with the restricted base station number through hybrid method. In this paper, hybrid localization method using the measured RSS and AOA data is suggested. The formulations of localization models based on RSS and AOA measurements are implemented separately and these two models are combined 
into the hybrid localization formula at the end of Section II.

\section{A. RSS Measurement Based Localization Model}

The relationship between the transmitted signal power from a target to $i$-th base station and the received signal power, denoted by $W_{t, i}$ and $W_{r, i}$, can be formulated as follows:

$$
W_{r, i}=K_{i} \frac{W_{t, i}}{d_{i}^{n}}
$$

where $K_{i}$ and $d_{i}$ denote the transmission parameter and the distance between a target and $i$-th base station, respectively. $K_{i}$ can be determined by considering antenna height, antenna gain, and received signal power. $n$ is the propagation constant that can be decided via obtaining the path loss slope by RSS measurements. $n$ can vary from 2 to 6 according to the transmission environment [9]. Based on (1), RSS value $r_{i}$ can be computed with the unknown target location $\mathbf{o}=\left[\begin{array}{ll}x & y\end{array}\right]^{T}$ and the known location of $i$-th base station $\mathbf{s}_{i}=\left[\begin{array}{ll}x_{i} & y_{i}\end{array}\right]^{T}, i=1, \cdots M$ as follows:

$$
\begin{aligned}
r_{i} & =K_{i} \frac{W_{t, i}}{W_{r, i}} \\
& =\left[\left(x-x_{i}\right)^{2}+\left(y-y_{i}\right)^{2}\right]^{\frac{n}{2}}
\end{aligned}
$$

Under the assumption that RSS value $r_{i}$ is correct in accordance with the distance, equation (2) can be satisfied with a following equation:

$$
x_{i} x+y_{i} y-0.5 R=\frac{1}{2}\left(x_{i}^{2}+y_{i}^{2}-r_{i}^{2 / n}\right)
$$

with $R=x^{2}+y^{2}$. The equation (3) can be expressed in matrix-vector form as follows:

$$
\mathbf{G}_{R S S} \boldsymbol{\theta}=\mathbf{Z}_{R S S}
$$

where

$$
\begin{aligned}
\mathbf{G}_{R S S} & =\left[\begin{array}{ccc}
x_{1} & y_{1} & -0.5 \\
\vdots & \vdots & \vdots \\
x_{M} & y_{M} & -0.5
\end{array}\right], \boldsymbol{\theta}=\left[\begin{array}{l}
x \\
y \\
R
\end{array}\right] \\
\mathbf{Z}_{R S S} & =\left[\begin{array}{c}
x_{1}^{2}+y_{1}^{2}-r_{1}^{2 / n} \\
\vdots \\
x_{M}^{2}+y_{M}^{2}-r_{M}^{2 / n}
\end{array}\right] .
\end{aligned}
$$

The vector $\boldsymbol{\theta}$ is the solution of the formulated RSS based localization model. In real case, the estimated location is different from the true location of a target since the parameter $\mathbf{Z}_{R S S}$ is affected by the measurement noise.

\section{B. AOA Measurement Based Localization Model}

The AOA data of the propagated wireless signal from a target measured by $i$-th base station can be formulated with the locations of a target and a base station as follows:

$$
\begin{aligned}
\tan \left(\sigma_{i}\right) & =\frac{\sin \left(\sigma_{i}\right)}{\cos \left(\sigma_{i}\right)} \\
& =\frac{y-y_{i}}{x-x_{i}}
\end{aligned}
$$

where $\sigma_{i}$ is the angle between the line of bearing from the $i$-th base station to a target and the $x$-axis. The equation (5) can be rewritten by rearranging as follows:

$$
x \sin \left(\sigma_{i}\right)-y \cos \left(\sigma_{i}\right)=x_{i} \sin \left(\sigma_{i}\right)-y_{i} \cos \left(\sigma_{i}\right)
$$

The matrix form of equation (6) can be acquired as a following equation.

$$
\mathbf{B}_{A O A} \mathbf{o}=\mathbf{Z}_{A O A}
$$

where

$$
\begin{aligned}
\mathbf{B}_{A O A} & =\left[\begin{array}{cc}
\sin \left(\sigma_{1}\right) & -\cos \left(\sigma_{1}\right) \\
\vdots & \vdots \\
\sin \left(\sigma_{M}\right) & -\cos \left(\sigma_{M}\right)
\end{array}\right], \\
\mathbf{Z}_{A O A} & =\left[\begin{array}{c}
x_{1} \sin \left(\sigma_{1}\right)-y_{1} \cos \left(\sigma_{1}\right) \\
\vdots \\
x_{M} \sin \left(\sigma_{M}\right)-y_{M} \cos \left(\sigma_{M}\right)
\end{array}\right] .
\end{aligned}
$$

The matrix $\mathbf{B}_{A O A}$ and the vector $\mathbf{Z}_{A O A}$ contain the noise factor since the AOA measurement $\sigma_{i}$ differs from the true angle of the propagation signal arrival under the real environment. An inaccuracy of localization is caused by these differences between a measurement and a true AOA data.

\section{RSS-AOA Hybrid Localization Model}

It is obvious that the combination of RSS and AOA measurements can improve the localization performance and reduce the number of required base stations. The hybrid localization equation using RSS and AOA measurements can be acquired by combining (4) and (7) as follows:

$$
\mathbf{G} \boldsymbol{\theta}=\mathbf{Z}
$$

where

$$
\begin{aligned}
& \mathbf{G}=\left[\begin{array}{l}
\mathbf{G}_{R S S} \\
\mathbf{G}_{A O A}
\end{array}\right], \mathbf{Z}=\left[\begin{array}{l}
\mathbf{Z}_{R S S} \\
\mathbf{Z}_{A O A}
\end{array}\right], \\
& \mathbf{G}_{A O A}=\left[\begin{array}{ll}
\mathbf{B}_{A O A} & \mathbf{0}
\end{array}\right] .
\end{aligned}
$$

In (8), the parameter $\mathbf{0}$ is an $M \times 1$ column vector in which all components are zeros. With $M$ base stations, the location of a target can be estimated precisely with $2 M$ 
measurement data set. However, the noise factors which are contained in parameters $\mathbf{G}$ and $\mathbf{Z}$ lead to localization inaccuracy. The optimal solution that minimizes the estimation error for a target localization using the Newton method is suggested in Section III.

\section{Newton Method BAsed Localization Solution}

The optimal solution minimizes the error between the estimated location and the true location of a target. To acquire the optimal solution, the objective function that means the amount of estimation error needs to be formulated at first. Since the noisy elements in matrix $\mathbf{G}$ and vector $\mathbf{Z}$ from equation (8) cause the estimation discrepancy, the objective function $f(\hat{\boldsymbol{\theta}})$ can be acquired as follows:

$$
f(\hat{\boldsymbol{\theta}})=(\mathbf{G} \hat{\boldsymbol{\theta}}-\mathbf{Z})^{T}(\mathbf{G} \hat{\boldsymbol{\theta}}-\mathbf{Z})
$$

with $\hat{\boldsymbol{\theta}}=\left[\begin{array}{lll}\hat{x} & \hat{y} & \hat{R}\end{array}\right]^{T}$ as an estimated design variable vector.

Among the various optimization methods, Newton method is a generalized scheme for finding better approximations to the roots of a differential equation. Newton method uses the fact that the tangent line to a curve is a good approximation to the curve near the point of tangency. The gradient $\mathbf{g}(\hat{\boldsymbol{\theta}})$ and the Hessian matrix $\mathbf{H}(\hat{\boldsymbol{\theta}})$ can express the tangent line to acquire the optimal solution that minimizes the objective function represented by equation (9). The gradient vector and the Hessian matrix can be acquired as follows:

$$
\begin{aligned}
\mathbf{g}(\hat{\boldsymbol{\theta}})= & {\left[\begin{array}{lll}
\frac{\partial f}{\partial \hat{x}} & \frac{\partial f}{\partial \hat{y}} & \frac{\partial f}{\partial \hat{R}}
\end{array}\right]^{T} } \\
= & \mathbf{G}^{T}(\mathbf{G} \hat{\boldsymbol{\theta}}-\mathbf{Z}) \\
\mathbf{H}(\hat{\boldsymbol{\theta}})= & {\left[\begin{array}{lll}
\frac{\partial^{2} f}{\partial \hat{x}^{2}} & \frac{\partial^{2} f}{\partial \hat{x} \partial \hat{y}} & \frac{\partial^{2} f}{\partial \hat{x} \partial \hat{R}} \\
\frac{\partial^{2} f}{\partial \hat{y} \partial \hat{x}} & \frac{\partial^{2} f}{\partial \hat{y}^{2}} & \frac{\partial^{2} f}{\partial \hat{y} \partial \hat{R}} \\
\frac{\partial^{2} f}{\partial \hat{R} \partial \hat{x}} & \frac{\partial^{2} f}{\partial \hat{R} \partial \hat{y}} & \frac{\partial^{2} f}{\partial \hat{R}^{2}}
\end{array}\right]=\mathbf{G}^{T} \mathbf{G} . }
\end{aligned}
$$

The initial point needs to be determined reasonably close to the true root in iteration process. Then the function that represents the tangent line of initial point can be obtained with the gradient vector and the Hessian matrix denoted by (10). The $x$-coordinates of the next point can be computed with $x$-intercept of the tangent line. The iteration solution of Newton method can be derived as follows:

$$
\hat{\boldsymbol{\theta}}_{a+1}=\hat{\boldsymbol{\theta}}_{a}+\rho\left[\mathbf{H}\left(\hat{\boldsymbol{\theta}}_{a}\right)\right]^{-1} \mathbf{g}\left(\hat{\boldsymbol{\theta}}_{a}\right) .
$$

where the step size $\rho \in(0,1]$ is determined experimentally. Then the Newton method based iteration process for acquiring optimal solution that minimizes the estimation error is as follows:

1) Set initial condition of $\hat{\boldsymbol{\theta}}_{a}$.

2) Compute the gradient $\mathbf{g}\left(\hat{\boldsymbol{\theta}}_{a}\right)$ and the Hessian matrix $\mathbf{H}\left(\hat{\boldsymbol{\theta}}_{a}\right)$. Acquire $\hat{\boldsymbol{\theta}}_{a+1}$ using equation (11).

3) If $\lambda=\left|f\left(\hat{\boldsymbol{\theta}}_{a}\right)-f\left(\hat{\boldsymbol{\theta}}_{a+1}\right)\right|$ is smaller than the threshold, stop the iteration. Otherwise, repeat $(a+1)$-th iteration through step 2) and 3).

\section{Simulation Results}

In Section IV, the reliability of the RSS-AOA measurement based hybrid localization scheme using Newton method is confirmed through some simulations. The RSS and AOA data of an active radio signal from a target is assumed to be measured for localization at five base stations of which the locations are $(0,0),(0,35),(50,0),(50,35)$, and $(100,0) \mathrm{km}$, respectively. The measurement errors of RSS and AOA data are assumed to follow the Gaussian distribution. The trajectory of the simulation has 210 time samples with one second interval. Moreover, the threshold parameter $\lambda$ of the iteration stop condition is supposed to be 0.01 in our simulations.

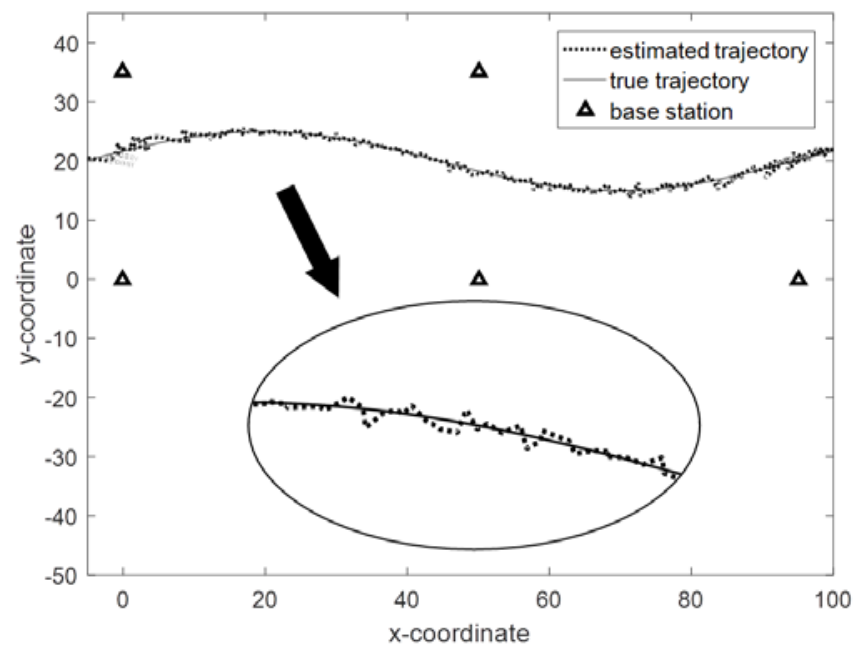

Fig. 1. The estimated trajectory of a target.

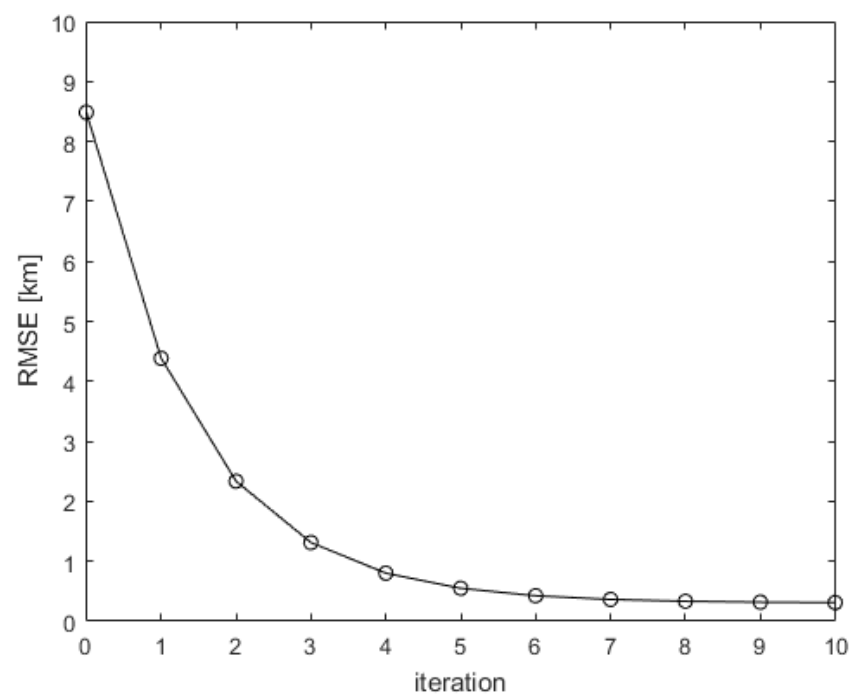

Fig. 2. RMSE changes with iteration process. 
Fig. 1 shows the estimated trajectory of a target using RSS-AOA based localization. The dotted line means the estimated trajectory and the solid line is the true trajectory of a target. The triangle symbol denotes the base stations. As shown in this figure, the estimated trajectory of a target using the proposed hybrid localization based on Newton method is almost the same with a true trajectory. In Fig. 2, the root mean square error (RMSE) changes of estimated target location with respect to the iteration process is demonstrated when the real location of a target is $(45,20) \mathrm{km}$. The RMSE of estimation converges to a minimized error which is calculated by the optimal solution of an objective function, denoted by equation (9).

\section{CONCLUSIONS}

The Newton method based optimal solution for hybrid localization model using RSS-AOA measurement was suggested in this paper. The hybrid localization model was formulated with the combination of respective RSS and AOA based localization model. RSS and AOA data measured from base stations include the measurement noise that causes the estimation inaccuracy of a target location. In order to obtain the precise location of a target, we formulated the objective function that represents the scalar error and obtained the optimal solution of this objective function through Newton method based iteration process. The localization performance of our proposed hybrid scheme was confirmed through some simulation results.

\section{ACKNOWLEDGMENT}

This work was supported by the National Research Foundation of Korea (NRF) grant funded by the Korea government (MSIP) (NRF-2016R1A2B4011369).

\section{REFERENCES}

[1] D. Shin and T. Sung, "Comparison of error characteristics between TOA and TDOA positioning," IEEE Transactions on Aerospace and Electronic Systems, vol. 38, pp. 307-311, January 2009.

[2] L. Cong and W. Zhuang, "Hybrid TDOA/AOA mobile user location for wideband CDMA cellular systems," IEEE Transactions on Wireless Communications, vol. 1, pp. 439-447, July 2002.

[3] D. Musicki, R. Kaune, and W. Koch, "Mobile emitter geolocation and tracking using TDOA and FDOA measurements," IEEE Transactions on Signal Processing, vol. 58, pp. 1863-1874, March 2010.
[4] P. Kulakowski, J. Alonso, E. Lopez, W. Ludwin, and J. Haro, “Angle of arrival localization based on antenna arrays for wireless sensor networks," Computer \& Electrical Engineering, vol. 36, pp. 1181-1186, November 2010.

[5] Q. Spencer, B. Jeffs, M. Jensen, and A. Swindlehurst, "Modeling the statistical time and angle of arrival characteristics of an indoor multipath channel," IEEE Journal on Selected Areas in Communications, vol. 18, pp. 347-360, March 2000.

[6] C. Nerguizian, C. Despins, and S. Affes, "Geolocation in mines with an impulse response fingerprinting technique and neural networks," IEEE Transactions on Wireless Communications, vol. 5, pp. 603-610, March 2006.

[7] G. Wang and H. Chen, "An importance sampling method for TDOA-based source localization,” IEEE Transactions on Wireless Communications, vol. 10, pp. 1560-1568, May 2011.

[8] S. Jung, S. Hann, and C. Park, "TDOA based optical wireless indoor localization using LED ceiling lamps," IEEE Transactions on Consumer Electronics, vol. 57, no. 4, pp. 1592-1597, November 2011.

[9] K. Cheung, H. So, W. Ma, and Y. Chan, "A constrained least squares approach to mobile positioning: algorithm and optimality,” EURASIP Journal on Applied Signal Processing, vol. 2006, pp. 1-23, December 2006.

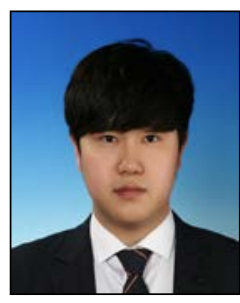

Hyukwoo Lee received his B.S. degree in electrical and computer engineering from Hankyung University in 2018. He is working as a research staff in applied optimization lab. at Sungkyunkwan University. His research interest fields are seismic wave discrimination with regression model and quadrotor tracking control using sliding-mode control.

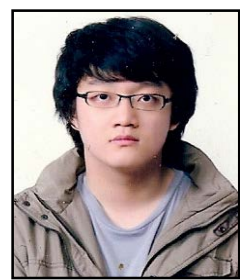

Kyunghyun Lee received his B.S. degree in electrical engineering from Sungkyunkwan University in 2013. He is working as a research staff in applied optimization lab. at Sungkyunkwan University. His current interest fields are intelligent control, geolocation problem with radio signal, measurement sensor development, estimation theory, and real-time nonlinear systems.

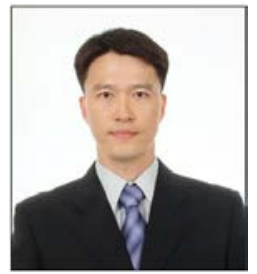

Kwanho You received his B.S. and M.S. degrees in electrical engineering from Korea Advanced Institute of Science and Technology (KAIST) in 1993 and 1996, and received his Ph.D. degree from the University of Minnesota in 2000 , respectively. In 2001, he joined the School of Information and Communication Engineering at Sungkyunkwan University. His research interests are wireless communication, adaptive optimization methods in nonlinear process, and estimation theory. 